N. E. Piskunov, W. W. Weiss, D. F. Gray, eds.

\title{
NLTE Radiative Transfer and Model Atmospheres of Hot Stars
}

\author{
Klaus Werner \\ Institut für Astronomie und Astrophysik, Universität Tübingen, Sand 1, \\ D-72076 Tübingen, Germany
}

\begin{abstract}
We describe our method to construct line blanketed NLTE model atmospheres for hot stars. We employ the Accelerated Lambda Iteration and use statistical methods to deal with metal line blanketing.
\end{abstract}

\section{Introduction}

Stellar atmospheres are open systems and thus cannot be in thermodynamic equilibrium (TE). The "Local Thermodynamic Equilibrium" (LTE) is a working hypothesis which assumes TE not for the atmosphere as a whole but for small volume elements. As a consequence, the atomic population numbers are depending only on the local (electron) temperature and electron density via the SahaBoltzmann equations. Computing models by replacing the Saha-Boltzmann equations by the rate equations (statistical equilibrium) are called non-LTE (or NLTE) models. NLTE calculations are more costly than LTE calculations, however, it is hard to predict if NLTE effects are important in a specific problem. Generally, NLTE effects are large at high temperatures and low densities, which implies intense radiation fields hence frequent radiative processes and less frequent particle collisions which tend to enforce LTE conditions.

We will restrict ourselves here to the classical model atmosphere problem, i.e. the solution of the radiation transfer equations assuming hydrostatic, radiative and statistical equilibrium. The numerical problem going from LTE to realistic NLTE models has been solved only recently and is the topic of this paper. This was achieved by the development of new numerical techniques for model construction and on the availability of atomic data for many species. The replacement of the Saha-Boltzmann equations by the atomic rate equations requires a different numerical solution technique, otherwise metal opacities cannot be accounted for at all. Such techniques were developed with big success during the last decade, triggered by important papers by Cannon (1973) and Scharmer (1981). The Accelerated Lambda Iteration (ALI) is at the heart of of this development. Combined with statistical methods we are finally able to compute metal line blanketed NLTE models with a very high level of sophistication.

\section{Overview of problem and solution method}

We assume plane-parallel geometry, which is well justified for most stars because the atmospheres are thin compared to the stellar radius. The only parameters 
which characterize uniquely such an atmosphere are the effective temperature $\left(T_{\text {eff }}\right)$, which is a measure for the amount of energy transported through the atmosphere per unit area and time, the surface gravity $(g)$, and the chemical composition. To construct model atmospheres we have to solve simultaneously a set of equations that is highly coupled and non-linear. Because of the coupling, no equation is determining uniquely a single quantity - all equations determine a number of state parameters. However, each of them is usually thought of as determining a particular quantity. These equations are:

- The radiation transfer equations which are solved for the (angular) mean intensities $J_{i}, i=1, \ldots, N F$, on a pre-chosen frequency grid comprising $N F$ points. The formal solution is given by $J=\Lambda S$, where $S$ is the source function (Eq. 10). Although $\Lambda$ is written as an operator, one may think of $\Lambda$ as a process of obtaining the mean intensity from the source function.

- The hydrostatic equation which determines the total particle density $N$.

- The radiative equilibrium equation from which the temperature $T$ follows.

- The particle conservation equation, determining the electron density $n_{e}$.

- The statistical equilibrium equations which are solved for the population densities $n_{i}, i=1, \ldots, N L$ of the atomic NLTE levels.

- The definition equation for a fictitious massive particle density $n_{H}$ which is introduced for a convenient representation of the solution procedure.

This set of equations has to be solved at each point $d$ of a grid comprising $N D$ depth points. Thus we are looking for solution vectors

$$
\boldsymbol{\psi}_{d}^{\prime}=\left(n_{1}, \ldots, n_{N L}, n_{e}, T, n_{H}, N, J_{1}, \ldots, J_{N F}\right), \quad d=1, \ldots, N D .
$$

The Complete Linearization (CL) method (Auer \& Mihalas 1969) solves this set by linearizing the equations with respect to all variables. The basic advantage of the ALI (or "operator splitting") method is that it allows to eliminate at the outset the explicit occurrence of the mean intensities $J_{i}$ from the solution scheme by expressing these variables by the current, yet to be determined, occupation densities and temperature. This is accomplished by an iteration procedure which may be written as (suppressing indices indicating depth and frequency dependency of variables):

$$
J^{n}=\Lambda^{\star} S^{n}+\left(\Lambda-\Lambda^{\star}\right) S^{n-1} .
$$

This means that the actual mean intensity at any iteration step $n$ is computed by applying an approximate lambda operator (ALO) $\Lambda^{\star}$ on the actual (thermal) source function $S^{n}$ plus a correction term that is computed from quantities known from the previous iteration step. This correction term includes the exact lambda operator $\Lambda$ which guarantees the exact solution of the radiation transfer problem in the limit of convergence: $J=\Lambda S$. The use of $\Lambda$ in Eq. 2 only indicates that a formal solution of the transfer equation is performed but in our application the operator is not constructed explicitly. Instead we employ the 
Feautrier solution scheme (Mihalas 1978) or a short characteristic method (Olson \& Kunasz 1987) to solve the transfer equation that is set up as a differential equation. The resulting set of equations for the reduced solution vectors

$$
\boldsymbol{\psi}_{d}=\left(n_{1}, \ldots, n_{N L}, n_{e}, T, n_{H}, N\right), \quad d=1, \ldots, N D
$$

is of course still non-linear. The solution is obtained by linearization and iteration which is performed either with a usual Newton-Raphson iteration or other methods (Sect. 5.2.). First model atmosphere calculations with the ALI method were performed by Werner (1986).

Another advantage of the ALI method is that explicit depth coupling of the solution vectors Eq. 1 through the transfer equation can be avoided if one restricts to diagonal (i.e. local) ALOs. Then the solution vectors Eq. 3 are independent from each other and the solution procedure within one iteration step of Eq. 2 is much more straightforward. Depth coupling is provided by the correction term that involves the exact solution of the transfer equation. The hydrostatic equation which also gives an explicit depth coupling, may be taken out of the set of equations and can - as experience shows - be solved in between two iteration steps of Eq. 2. Then full advantage of a local ALO can be taken. The linearized system may be written as:

$$
\boldsymbol{\psi}_{d}=\boldsymbol{\psi}_{d}^{0}+\delta \boldsymbol{\psi}_{d}
$$

where $\boldsymbol{\psi}_{d}^{0}$ is the current estimate for the solution vector at depth $d$ and $\delta \boldsymbol{\psi}_{d}$ is the correction vector to be computed. Using a tri-diagonal $\Lambda^{\star}$ operator the resulting system for $\delta \psi_{d}$ is - like in the classical CL scheme - of block tri-diagonal form coupling each depth point $d$ to its nearest neighbors $d \pm 1$ :

$$
\gamma_{d} \delta \psi_{d-1}+\boldsymbol{\beta}_{d} \delta \psi_{d}+\boldsymbol{\alpha}_{d} \delta \psi_{d+1}=\mathbf{c}_{d}
$$

The quantities $\boldsymbol{\alpha}, \boldsymbol{\beta}, \boldsymbol{\gamma}$ are $(N N \times N N)$ matrices where $N N$ is the total number of physical variables, i.e., $N N=N L+4$, and $\mathbf{c}_{d}$ is the residual error in the equations. The solution is obtained by the standard Feautrier scheme. As already mentioned, the system Eq. 5 breaks into $N D$ independent equations $\delta \boldsymbol{\psi}_{d}=\boldsymbol{\beta}_{d}^{-1} \mathbf{c}_{d}(d=1, \ldots, N D)$ when a local $\Lambda^{\star}$ operator is used. The additional numerical effort to set up the subdiagonal matrices and matrix multiplications in the tri-diagonal case is outweighed by the faster global convergence of the ALI cycle, accomplished by the explicit depth coupling in the linearization procedure (Werner 1989).

The principal advantage of the ALI over the CL method becomes clear at this point. Each matrix inversion necessary to solve Eq. 5 requires $(N L+4)^{3}$ operations whereas in the CL method $(N L+N F+4)^{3}$ operations are needed. Since the number of frequency points $N F$ is much larger than the number of levels $N L$, the matrix inversion in the CL approach is dominated by $N F$.

Recent developments concern the problem that the total number of atomic levels tractable in NLTE with the ALI method described so far is restricted to the order of 250, from experience with our model atmosphere code PR02. This limit is a consequence of the non-linearity of the equations, and in order to overcome it, measures must be taken in order to achieve a linear system whose numerical solution is much more stable. Such a pre-conditioning procedure has been first applied in the ALI context by Werner \& Husfeld (1985) using the core 
saturation method (Rybicki 1972). More advanced work achieves linearity by replacing the $\Lambda$ operator with the $\Psi$ operator (and by judiciously considering some populations as "old" and some as "new" ones within an ALI step) which is formally defined by writing:

$$
J_{\nu}=\Psi_{\nu} \eta_{\nu}, \quad \text { i.e. } \quad \Psi_{\nu} \equiv \Lambda_{\nu} / \chi_{\nu}
$$

where the total opacity $\chi_{\nu}$ (as defined in Sect. 3.6.) is calculated from the previous ALI cycle. The advantage is that the emissivity $\eta_{\nu}$ (Sect. 3.6.) is linear in the populations, whereas the source function $S_{\nu}$ is not. Hence the new operator $\Psi$ gives the solution of the transfer problem by acting on a linear function. This idea is based on Rybicki \& Hummer (1991) who applied it to the line formation problem, i.e. restricting the set of equations to the transfer and rate equations and regarding the atmospheric structure as fixed. Hauschildt (1993) and Hauschildt \& Baron (1999) generalized it to solve the full model atmosphere problem. In addition, splitting the set of statistical equations and solving it separately for each chemical element means that now many hundreds of levels per species are tractable in NLTE.

\section{Basic equations}

We give a short overview of the basic equations to be solved. More detailed presentations may be found in Werner \& Dreizler (1999) or Werner et al. (2003).

\subsection{Radiation transfer}

Any numerical method requires a formal solution (i.e. atmospheric structure already given) of the radiation transfer problem. The radiation transfer at any particular depth point can be described by the following equation, formally written for positive and negative $\mu$ (which is the cosine of the angle between direction of propagation and outward directed normal to the surface) separately, i.e. for inward and for outward directional intensities $I$ with frequency $\nu$ :

$$
\pm \mu \frac{\partial I_{\nu}( \pm \mu)}{\partial \tau_{\nu}}=S_{\nu}-I_{\nu}( \pm \mu), \quad \mu \in[0,1] .
$$

$\tau_{\nu}$ is the optical depth (which can be defined via the column mass $m$ that is used in the other structural equations by $d \tau_{\nu}=d m \chi_{\nu} / \rho$, with the mass density $\rho$ ) and $S_{\nu}$ is the local source function. Introducing the Feautrier intensity

$$
u_{\nu \mu} \equiv\left(I_{\nu}(\mu)+I_{\nu}(-\mu)\right) / 2
$$

we obtain the second-order form (Mihalas 1978):

$$
\mu^{2} \frac{\partial^{2} u_{\nu \mu}}{\partial \tau_{\nu}^{2}}=u_{\nu \mu}-S_{\nu}, \quad \mu \in[0,1]
$$

We may separate the Thomson emissivity term (scattering from free electrons, assumed coherent, with cross-section $\sigma_{e}$ ) from the source function so that

$$
S_{\nu}=S_{\nu}^{\prime}+n_{e} \sigma_{e} J_{\nu} / \chi_{\nu}
$$


where $S_{\nu}^{\prime}$ is the ratio of thermal emissivity to total opacity (Sect. 3.6.): $S_{\nu}^{\prime}=$ $\eta_{\nu} / \chi_{\nu}$. Since the mean intensity is the angular integral over the Feautrier intensity the transfer equation becomes

$$
\mu^{2} \frac{\partial^{2} u_{\nu \mu}}{\partial \tau_{\nu}^{2}}=u_{\nu \mu}-S_{\nu}^{\prime}-\frac{n_{e} \sigma_{e}}{\chi_{\nu}} \int_{0}^{1} u_{\nu \mu} d \mu
$$

Thomson scattering complicates the situation by explicit angle coupling. Assuming complete frequency redistribution in spectral lines, no explicit frequency coupling occurs so that the parallel solution for all frequencies enables a very efficient vectorization on the computer.

\subsection{Statistical equilibrium}

Rate equations For each atomic level $i$ the rate equation describes the equilibrium of rates into and rates out of this level:

$$
n_{i} \sum_{i \neq j} P_{i j}-\sum_{j \neq i} n_{j} P_{j i}=0 .
$$

The rate coefficients $P_{i j}$ have radiative and collisional components: $P_{i j}=R_{i j}+$ $C_{i j}$. The radiative downward rate for example is given by:

$$
R_{j i}=\left(\frac{n_{i}}{n_{j}}\right)^{\star} 4 \pi \int_{0}^{\infty} \frac{\sigma_{i j}(\nu)}{h \nu}\left(\frac{2 h \nu^{3}}{c^{2}}+J_{\nu}\right) e^{-h \nu / k T} d \nu
$$

Photon cross-sections are denoted by $\sigma_{i j}(\nu) .\left(n_{i} / n_{j}\right)^{\star}$ is the Boltzmann LTE population ratio. The computation of collisional rates is generally dependent on the specific ion or even transition.

Abundance definition equation The rate equation for the highest level of a given chemical species is redundant. It is replaced by the abundance definition equation. Summation over all levels usually includes not only NLTE levels but also levels which are treated in LTE, according to the specification in the model atom. Denoting the number of ionization stages of species $k$ with $N I O N(k)$, the number of NLTE and LTE levels per ion with $N L(l)$ and $L T E(l)$, respectively, and the number fraction of species $k$ with $y_{k}$, we can write:

$$
\sum_{l=1}^{N I O N(k)}\left[\sum_{i=1}^{N L(l)} n_{k l i}+\sum_{i=1}^{L T E(l)} n_{k l i}^{\star}\right]=y_{k}\left(N-n_{e}\right) .
$$

Charge conservation We close the system of statistical equations by invoking charge conservation. We denote the total number of chemical species with NATOM, the charge of ion $l$ with $q(l)$ (in units of the electron charge) and write:

$$
\sum_{k=1}^{N A T O M} \sum_{l=1}^{N I O N(k)} q(l)\left[\sum_{i=1}^{N L(l)} n_{k l i}+\sum_{i=1}^{L T E(l)} n_{k l i}^{\star}\right]=n_{e} .
$$


Complete statistical equilibrium equations We introduce a vector comprising the occupation numbers of all NLTE levels, $\mathbf{n}=\left(n_{1}, \ldots, n_{N L}\right)$. Then the statistical equilibrium equation is written as:

$$
\mathbf{A n}=\mathbf{b} .
$$

The gross structure of the rate matrix $\mathbf{A}$ is of block matrix form, because transitions between levels occur within one ionization stage or to the ground state of the next ion. The structure is complicated by ionizations into excited levels and by the abundance definition and charge conservation equations which give additional non-zero elements in the corresponding lines of $\mathbf{A}$.

\subsection{Radiative equilibrium}

Radiative equilibrium can be enforced by adjusting the temperature stratification either during the linearization procedure or in between ALI iterations. In the former case a linear combination of two different formulations can be used and in the latter case the classical Unsöld-Lucy temperature correction procedure (Lucy 1964) is utilized. The latter is particularly interesting, because it allows to exploit the blocked form of the rate coefficient matrix. This will enable an economic block-by-block solution followed by a subsequent Unsöld-Lucy temperature correction step. On the other side, however, this correction procedure may decelerate the global convergence behavior of the ALI iteration.

The two forms of expressing the radiative equilibrium condition follow from the postulation that the energy emitted by a volume element per unit time is equal to the absorbed energy per unit time (integral form):

$$
\int_{0}^{\infty} \chi_{\nu}\left(S_{\nu}-J_{\nu}\right) d \nu=0
$$

This formulation is equivalent to invoking flux constancy throughout the atmosphere (differential form) involving the nominal flux $\mathcal{H}$ :

$$
\int_{0}^{\infty} \frac{\partial}{\partial \tau_{\nu}}\left(f_{\nu} J_{\nu}\right) d \nu-\mathcal{H}=0
$$

where $f_{\nu}$ is the variable Eddington factor, defined as

$$
f_{\nu}=\int_{0}^{1} \mu^{2} u_{\nu \mu} d \mu / \int_{0}^{1} u_{\nu \mu} d \mu
$$

and computed from the Feautrier intensity $u_{\nu \mu}$ (Eq. 8) after the formal solution. The differential form is more accurate at large depths, while the integral form behaves numerically better at small depths. Instead of arbitrarily selecting that depth in the atmosphere where we switch from one formulation to the other, we use a linear combination of both constraint equations which guarantees a smooth transition with depth, based on physical grounds:

$$
\frac{1}{\bar{\kappa}_{J}} \int_{0}^{\infty} \chi_{\nu}\left(S_{\nu}-J_{\nu}\right) d \nu+\bar{\Lambda}_{J}^{\star} \int_{0}^{\infty} \frac{\partial}{\partial \tau_{\nu}}\left(f_{\nu} J_{\nu}\right) d \nu-\bar{\Lambda}_{J}^{\star} \mathcal{H}-F_{0}=0 .
$$

For details on this equation and on our implementation of the Unsöld-Lucy procedure see Dreizler (2003). 


\subsection{Hydrostatic equilibrium}

The total pressure $P$ comprises gas, radiation and turbulent pressures, so that we write the equation for hydrostatic equilibrium as:

$$
\frac{d}{d m} P=\frac{d}{d m}\left(N k T+\frac{4 \pi}{c} \int_{0}^{\infty} f_{\nu} J_{\nu} d \nu+\frac{1}{2} \rho v_{\text {turb }}^{2}\right)=g
$$

with Boltzmann's constant $k$ and the turbulent velocity $v_{\text {turb }}$. The hydrostatic equation may either be solved simultaneously with all other equations or separately in between iterations. The overall convergence behavior is usually the same in both cases. If taken into the linearization scheme and a local $\Lambda^{\star}$ operator is used then, like in the case of the radiative equilibrium equation, explicit depth coupling enters via the depth derivative $d / d m$. Again, solution of the linearized equations has to proceed inwards starting at the outer boundary.

\subsection{Particle conservation and fictitious massive particle density}

The total particle density $N$ is the sum of electron density plus the population density of all atomic states, LTE and NLTE levels:

$$
N=n_{e}+\sum_{k=1}^{N A T O M} \sum_{l=1}^{N I O N(k)}\left[\sum_{i=1}^{N L(l)} n_{k l i}+\sum_{i=1}^{L T E(l)} n_{k l i}^{\star}\right]
$$

A fictitious massive particle density $n_{H}$ is defined by:

$$
n_{H}=\sum_{k=1}^{N A T O M} m_{k} \sum_{l=1}^{N I O N(k)}\left[\sum_{i=1}^{N L(l)} n_{k l i}+\sum_{i=1}^{L T E(l)} n_{k l i}^{\star}\right]
$$

The mass of a chemical species in AMU is denoted by $m_{k}$. Introducing the mass of a hydrogen atom $m_{H}$, we then may simply write for the material density

$$
\rho=n_{H} m_{H}
$$

\subsection{Opacity and emissivity}

Thermal opacity and emissivity are made up by radiative bound-bound, boundfree and free-free transitions. For each species we compute and sum up:

$$
\begin{aligned}
\kappa_{\nu}= & \sum_{l=1}^{N I O N}\left[\sum_{i=1}^{N L(l)} \sum_{j>i}^{N L(l)} \sigma_{l i \rightarrow l j}(\nu)\left(n_{l i}-n_{l j} \frac{g_{l i}}{g_{l j}} e^{-h\left(\nu-\nu_{i j}\right) / k T}\right)\right. \\
& +\sum_{i=1}^{N L(l)} \sum_{j>i}^{N L(l+1)} \sigma_{l i \rightarrow l+1, k}(\nu)\left(n_{l i}-n_{l i}^{\star} e^{-h \nu / k T}\right) \\
& \left.+n_{e} \sigma_{k k}(l, \nu)\left(1-e^{-h \nu / k T}\right)\left(\sum_{i=1}^{N L(l+1)} n_{l+1, i}+\sum_{i=1}^{L T E(l+1)} n_{l+1, i}^{\star}\right)\right]
\end{aligned}
$$


where the total opacity includes Thomson scattering, i.e. $\chi_{\nu}=\kappa_{\nu}+n_{e} \sigma_{e}$, and

$$
\begin{aligned}
\frac{\eta_{\nu}}{2 h \nu^{3} / c^{2}}= & \sum_{l=1}^{N I O N}\left[\sum_{i=1}^{N L(l)} \sum_{j>i}^{N L(l)} \sigma_{l i \rightarrow l j}(\nu) n_{l j} \frac{g_{l i}}{g_{l j}} e^{-h\left(\nu-\nu_{i j}\right) / k T}\right. \\
& +\sum_{i=1}^{N L(l)} \sum_{j>i}^{N L(l+1)} \sigma_{l i \rightarrow l+1, k}(\nu) n_{l i}^{\star} e^{-h \nu / k T} \\
& \left.+n_{e} \sigma_{k k}(l, \nu) e^{-h \nu / k T}\left(\sum_{i=1}^{N L(l+1)} n_{l+1, i}+\sum_{i=1}^{L T E(l+1)} n_{l+1, i}^{\star}\right)\right] .
\end{aligned}
$$

$\sigma_{l i \rightarrow l+1, k}(\nu)$ denotes the cross-section for photoionization from level $i$ of ion $l$ into level $k$ of ion $l+1$. The double summation over the bound-free continua takes into account the possibility that a particular level may be ionized into more than one level of the next high ion. The source function used for the approximate radiation transfer is $\eta_{\nu} / \kappa_{\nu}$, thus, excludes Thomson scattering. For the exact formal solution of course, the total opacity $\chi_{\nu}$ in the expression Eq. 10 includes the Thomson term $\left(n_{e} \sigma_{e}\right)$.

\section{The Accelerated Lambda Iteration (ALI)}

In all constraint equations described above the mean intensities $J_{\nu}$ are substituted by the approximate radiation field Eq. 2 in order to eliminate these variables from the solution vector Eq. 1 . In principle the ALO may be of arbitrary form as long as the iteration procedure converges. In practice however an optimum choice is desired in order to achieve convergence with a minimum amount of iteration steps.

In the case of diagonal (local) lambda operators the mean intensity $J_{d}$ at a particular depth $d$ in the current iteration step is computed solely from the local source function $S_{d}$ and a correction term $\Delta J_{d}$, the latter involving the source functions (of all depths) from the previous iteration. Dropping the iteration count and introducing indices denoting depth points we can rewrite Eq. 2:

$$
J_{d}=\Lambda_{d, d}^{\star} S_{d}+\Delta J_{d}
$$

In the discrete form we now think of $\Lambda^{\star}$ as a matrix acting on a vector whose elements comprise the source functions of all depths. Then $\Lambda_{d, d}^{\star}$ is the diagonal element of the $\Lambda^{\star}$ matrix corresponding to depth point $d$. Writing $\Lambda_{d, d}^{\star} \equiv B_{d}$ we have a purely local expression for the mean intensity:

$$
J_{d}=B_{d} S_{d}+\Delta J_{d}
$$

Much better convergence is obtained if the mean intensity is computed not only from the local source function but also from the source function of the neighboring depths points. Then the matrix representation of $\Lambda^{\star}$ is of tri-diagonal form and we may write:

$$
J_{d}=C_{d-1} S_{d-1}+B_{d} S_{d}+A_{d+1} S_{d+1}+\Delta J_{d}
$$


where $C_{d-1}$ and $A_{d+1}$ represent the upper and lower subdiagonal elements of $\Lambda^{\star}$ and $S_{d \pm 1}$ the source functions at the adjacent depths. We emphasize again that the actual source functions in Eq. 29 are computed from the actual population densities and temperature which are unknown. We therefore have a non-linear set of equations which is solved by either a Newton-Raphson iteration or other techniques, resulting in the solution of a tri-diagonal linear equation of the form Eq. 5. It was shown in Olson et al. (1986) that the elements of the optimum $\Lambda^{\star}$ matrix are given by the corresponding elements of the exact $\Lambda$ matrix.

\section{Solution of the non-linear equations by iteration}

The complete set of non-linear equations for a single iteration step Eq. 2 comprises at each depth the equations for statistical, radiative, and hydrostatic equilibrium and the particle conservation equation. For the numerical solution we introduce discrete depth and frequency grids. The equations are then linearized and solved by a suitable iterative scheme. Explicit angle dependency of the radiation field is not required here and consequently eliminated by the use of variable Eddington factors. Angle dependency is only considered in the formal solution of the transfer equation.

\subsection{Discretization and Linearization}

A depth grid is set up and we start from a gray approximation computing a LTE continuum model using the Unsöld-Lucy temperature correction procedure. Depth points (typical number is 90 ) are set equidistantly on a logarithmic (Rosseland) optical depth scale. Our NLTE code uses the column mass as an independent depth variable.

The frequency grid is established based upon the atomic data input file. Frequency points are set blue- and redward of each absorption edge and for each spectral line. Gaps are filled in by setting continuum points. Finally, the quadrature weights are computed. Frequency integrals appearing e.g. in Eq. 20 are replaced by quadrature sums and differential quotients involving depth derivatives by difference quotients.

All variables $x$ are replaced by $x \rightarrow x+\delta x$ where $\delta x$ denotes a small perturbation of $x$. Terms not linear in these perturbations are neglected. The perturbations are expressed by perturbations of the basic variables:

$$
\delta x=\frac{\partial x}{\partial T} \delta T+\frac{\partial x}{\partial n_{e}} \delta n_{e}+\frac{\partial x}{\partial N} \delta N+\frac{\partial x}{\partial n_{H}} \delta n_{H}+\sum_{l=1}^{N L} \frac{\partial x}{\partial n_{l}} \delta n_{l} .
$$

\subsection{Newton-Raphson iteration and alternative methods}

As described in Sect. 2. the linearized equations have a tri-diagonal block-matrix form, see Eq. 5. Inversion of the grand matrix ( $\equiv \mathbf{T}$ sized $(N N \cdot N D) \times(N N$. $N D$ ), i.e. about $10^{4} \times 10^{4}$ in typical applications) is performed with a blockGaussian elimination scheme, which means that our iteration of the non-linear equations represents a multi-dimensional Newton-Raphson method. The problem is structurally simplified when explicit depth coupling is avoided by the use of a local ALO, however, the numerical effort is not much reduced, because in 
both cases the main effort lies with the inversion of matrices sized $N N \times N N$. The Newton-Raphson iteration involves two numerically expensive steps, first setting up the Jacobian (comprising $\boldsymbol{\alpha}, \boldsymbol{\beta}, \boldsymbol{\gamma}$ ) and then inverting it. Additionally, the matrix inversions necessary to solve Eq. 5 limit their size to about $N N=250$ because otherwise numerical accuracy is lost.

Two variants recently introduced in stellar atmosphere calculations are able to improve both, numerical accuracy and, most of all, computational speed. Broyden's (1965) variant belongs to the family of quasi-Newton methods and it was first used in model atmosphere calculations in Dreizler \& Werner (1991), Hamann et al. (1991) and Koesterke et al. (1992). It avoids the repeated set-up of the Jacobian by the use of an update formula. On top of this, it also gives an update formula for the inverse Jacobian. Another variant, the Kantorovich method was introduced into model atmosphere calculations by Hubeny \& Lanz (1992). This method simply keeps fixed the Jacobian during the linearization cycle and it is surprisingly stable.

\section{NLTE metal line blanketing}

Despite the capacity increase for the NLTE treatment of model atmosphere problems by introducing the ALI method combined with pre-conditioning techniques, the blanketing by millions of lines from the iron group elements arising from transitions between some $10^{5}$ levels could only be attacked with the help of statistical methods. These have been introduced into NLTE model atmosphere work by Anderson (1989). At the outset, model atoms are constructed by combining many thousands of levels into a relatively small number of superlevels which can be treated with ALI (or other) methods. Then, in order to reduce the computational effort, two approaches were developed which vastly decrease the number of frequency points (and hence the number of transfer equations to be solved) to describe properly the complex frequency dependence of the opacity. These two approaches have their roots in LTE modeling techniques, where for the same reason statistical methods are applied for the opacity treatment: The Opacity Distribution Function (ODF) and Opacity Sampling (OS) approaches. Both are based on the circumstance that the opacity (in the LTE approximation) is a function of two only local thermodynamic quantities. Roughly speaking, each opacity source can be written in terms of a population density and a photon cross-section for the respective radiative transition: $\kappa_{\nu} \sim n_{l} \sigma_{l u}(\nu)$. In LTE the population follows from the Saha-Boltzmann equations, hence $n_{l}=n_{l}\left(n_{e}, T\right)$. The OS and ODF methods use such pre-tabulated (on a very fine frequency mesh) $\kappa_{\nu}\left(n_{e}, T\right)$ during the model atmosphere calculations. The NLTE situation is more complicated, because pre-tabulation of opacities is not useful. The population densities at any depth now also depend explicitly on the radiation field (via the rate equations which substitute the TE Saha-Boltzmann statistics) and thus on the populations in each other depth of the atmosphere. As a consequence, the OS and ODF methods are not applied to opacity tabulations, but on tabulations of the photon cross-sections $\sigma(\nu)$. These do depend on local quantities only, e.g. line broadening by Stark and Doppler effects is calculated from $T$ and $n_{e}$. In the NLTE case the cross-section takes over the role which 
the opacity played in the LTE case. More details on this topic can be found in Werner \& Dreizler (1999).

Our strategy is the following. Before any model atmosphere calculation is started, the atomic data are prepared by constructing superlevels, and the cross-sections for superlines. Then these cross-sections are either sampled on a coarse frequency grid or ODFs are constructed. These data are put into the model atom which is read by the model code.

\section{Summary}

The construction of metal line blanketed models in hydrostatic and radiative equilibrium under NLTE conditions was the last and long-standing problem of classical model atmosphere theory and it is finally solved with a high degree of sophistication. The essential milestones for this development, starting from the pioneering work of Auer \& Mihalas (1969) are:

- Introduction of the Accelerated Lambda Iteration (ALI, or "operator splitting" methods), based upon early work by Cannon (1973) and Scharmer (1981). First ALI model atmospheres were constructed by Werner (1986).

- Introduction of statistical approaches to treat the iron group elements in NLTE by Anderson (1989).

- Linear formulation of the statistical equations (Rybicki \& Hummer 1991, Hauschildt 1993).

- Computation of atomic data by Kurucz (1991), by the Opacity Project (Seaton et al. 1994) and the Iron Project (Hummer et al. 1993).

Acknowledgments. Stellar atmosphere work in Tübingen is supported by DFG and DLR. I thank for financial support from the conference organizers.

\section{Discussion}

SAPAR: Usual Lambda Iteration in physical terms is iteration according to the order of multiple scattering. What is the accelerated Lambda Iteration in physical terms?

WERNER: The accelerated Lambda Iteration basically avoids following the multiple scattering of photons, e.g. in optically thick line cores. This is sensible, because those photons do not travel far between two scattering events. The ALI does this based on physical grounds (core saturation) and in a correct (not approximate) way.

STEFFEN: What kind of assumptions is made for you accretion disk models? Is plane-parallel geometry justified?

WERNER: The disk is divided in a number of concentric rings. Plane-parallel geometry is assumed for each individual ring. The radial disk structure is calculated according to the theory of Shakura \& Sunyaev. Our calculations concentrate on a detailed NLTE-modelling of the vertical disk structure and the emergent spectrum. 


\section{References}

Anderson, L. S. 1989, ApJ, 339, 558

Auer, L. H., \& Mihalas, D. 1969, ApJ, 158, 641

Broyden, C. G. 1965, Math. Comp., 19, 577

Cannon, C. J. 1973, ApJ, 185, 621

Dreizler, S. 2003, in Stellar Atmosphere Modeling, ed. I. Hubeny, D. Mihalas, \& K. Werner, ASP Conf. Series, in press

Dreizler, S., \& Werner, K. 1991, in Stellar Atmospheres: Beyond Classical Models, NATO ASI Series C, Vol. 341, ed. L. Crivellari, I. Hubeny \& D.G. Hummer, (Dordrecht: Kluwer), 155

Hamann, W.-R., Koesterke, L., \& Wessolowski, U. 1991, in Stellar Atmospheres: Beyond Classical Models, NATO ASI Series C, Vol. 341, ed. L. Crivellari, I. Hubeny \& D.G. Hummer, (Dordrecht: Kluwer), 69

Hauschildt, P. H. 1993, JQSRT, 50, 301

Hauschildt, P. H., \& Baron, E. 1999, in Computational Astrophysics, ed. H. Riffert \& K. Werner, J. of Comp. and Applied Mathematics, 109, 41

Hubeny, I., \& Lanz, T. 1992, A\&A, 262, 501

Hummer, D. G., Berrington, K. A., Eissner, W., Pradhan, A. K., Saraph, H. E., \& Tully, J. A. 1993, A\&A, 279, 298

Koesterke, L., Hamann, W.-R., \& Kosmol, P. 1992, A\&A, 255, 490

Kurucz, R. L. 1991, in Stellar Atmospheres: Beyond Classical Models, NATO ASI Series C, Vol. 341, ed. L. Crivellari, I. Hubeny \& D.G. Hummer, (Dordrecht: Kluwer), 441

Lucy, L. B. 1964, in First Harvard Smithsonian Conference on Stellar Atmospheres, SAO Special Report No. 167, Cambridge, Massachusetts, 93

Mihalas, D. 1978, Stellar Atmospheres, 2nd edition, (San Francisco: Freeman)

Olson, G. L., \& Kunasz, P. B. 1987, JQSRT, 38, 325

Olson, G. L., Auer, L. H., \& Buchler, J. R. 1986, JQSRT, 35, 431

Rybicki, G. B. 1972 in Line Formation in the Presence of Magnetic Fields, ed. R. G. Athay, L. House \& G. Newkirk, Jr. (Boulder: High Altitude Observatory), 145

Rybicki, G. B., \& Hummer, D. G. 1991, A\&A, 245, 171

Scharmer, G. B. 1981, ApJ, 249, 720

Seaton, M. J., Yan, Y., Mihalas, D., \& Pradhan, A. K. 1994, MNRAS, 266, 805

Werner, K. 1986, A\&A, 161, 177

Werner, K. 1989, A\&A, 226, 265

Werner, K., \& Husfeld, D. 1985, A\&A, 148, 417

Werner, K., \& Dreizler, S. 1999, in Computational Astrophysics, ed. H. Riffert \& K. Werner, J. of Comp. and Applied Math., 109, 65

Werner, K., Deetjen, J.L., Dreizler, S., Nagel, T., Rauch, T., \& Schuh, S.L. 2003 in Stellar Atmosphere Modeling, ed. I. Hubeny, D. Mihalas, \& K. Werner, ASP Conf. Series, in press 